\title{
Aspergillus niveus
}

National Cancer Institute

\section{Source}

National Cancer Institute. Aspergillus niveus. NCI Thesaurus. Code C150877.

A species of mold fungus in the phylum Ascomycota that is commonly found in soil. This species is a rare opportunistic pathogen in humans. 